\title{
$\bullet$ Radiation-Induced Dysphagia and Life-Threatening Stridor in Head and Neck Cancer: A Review
}

\section{IJCRR}

Section: Healthcare

ISI Impact Factor

(2019-20): 1.628

IC Value (2019): 90.81 $\operatorname{SJIF}(2020)=7.893$

(c) (7) (3)

Copyright@IJCRR

\section{Santosh Kumar Swain, Satyabrata Acharya, Smarita Lenka}

Department of Otorhinolaryngology, IMS and SUM Hospital, Siksha “O” Anusandhan University, K8, Kalinganagar, Bhubaneswar-751003, Odisha, India.

\section{ABSTRACT}

Radiotherapy plays an important role in the treatment of head and neck cancer. Radiation therapy to the head and neck cancer can cause an uncommon long term swallowing and breathing difficulties by causing stenosis at the pharynx, larynx and oesophagus. The dysphagia or breathing difficulties by radiation therapy may occur be mechanical, structural and neurological deficits. It hurts the quality of life among cancer survivors. This acquired pharyngeal stenosis after radiotherapy may threaten to live by compromising the airway. Radiation-induced pharyngeal stenosis may occur in cases with head and neck cancer which cause significant morbidity and mortality. This article reviews details of radiation-induced pharyngeal or upper aerodigestive stenosis with manifestations of dysphagia and compromised airway including its epidemiology, pathophysiology, clinical presentations, investigations and possible treatment options. The pathogenesis for dysphagia and stridor due to pharyngeal and oesophagal stenosis following radiotherapy is unclear. Severe mucositis in the pharynx might be the predisposing factor for causing fibrosis and leads to stenosis. The upper aerodigestive tract stenosis following radiotherapy is an unusual complication which can be treated with dilation or excision with scar tissue. Although there is no specific or gold standard treatment option found for this stenosis, many supportive, restorative and palliative treatments are available under various clinical conditions.

Key Words: Pharyngeal stenosis, Radiotherapy, Dysphagia, Stridor

\section{INTRODUCTION}

Radiotherapy is an important treatment option in head and neck squamous cell carcinoma. Along with surgery or chemotherapy, radiotherapy plays an important role in the treatment of the malignancy in the head and neck region. ${ }^{1}$ Many surgeons prefer preoperative radiotherapy while some choose planned postoperative radiation. In some patients, full therapeutic doses of radiotherapy are used, although in a reduced dose. The pharyngeal or oesophagal stenosis may be found as a complication of radiation exposure of the head and neck cancer. These manifestations can cause morbidity and life-threatening situation among the cancer-free survivors. Nasopharyngeal carcinoma is an important head and neck cancer where the treatment of choice is radiotherapy. ${ }^{2}$ There are several associated complications following radiotherapy to the head and neck region such as endocrine dysfunction and temporal lobe injury other than synechia the pharynx and oesophagus. ${ }^{3}$ The uncommon and dreaded complications of radiotherapy such as dysphagia and stridor are due to fibrosis and narrowing at the pharynx and oesophagus following radiotherapy. These clinical entities are less reported in the medical literature. The delayed effects of the radiation on the pharynx, oesophagus and larynx have not been studied adequately but these parts are often exposed to the radiation during the time of treatment of the head and neck cancer like nasopharyngeal carcinoma. The stenosis at the hypopharynx or upper oesophagus affects the swallowing at the initial period. However, in severe stenosis, it can lead to stridor along with dysphagia to the solid foods. This review article summarizes the epidemiology, pathophysiology, clinical manifestations, investigations and current treatment of the radiation-induced dysphagia and stridor.

\section{MATERIALS AND METHODS}

For searching the published research articles, we conducted an electronic survey of the SCOPUS, Medline, Google Scholar and Pubmed database (Figure 1). A search strategy

\section{Corresponding Author:}

Dr. Santosh Kumar Swain, Professor, Department of Otorhinolaryngology, IMS\&SUM Hospital, Siksha “O” Anusandhan University, K8, Kalinganagar, Bhubaneswar-751003, Odisha, India; Cell: +91-9556524887; Email: santoshswain@soa.ac.in

ISSN: 2231-2196 (Print)

Received: 21.09 .2020
ISSN: 0975-5241 (Online)

Revised: 08.11 .2020
Accepted: 12.12 .2020
Published: 03.03 .2021 
using PRISMA (Preferred Reporting Items for Systematic Reviews and Meta-Analysis) guidelines was developed (Fig. 1). Randomized controlled studies, observational studies, comparative studies, case series and case reports were evaluated for the eligibility. This review paper focuses only on the radiation-induced dysphagia and stridor. The search articles with no primary data related to the radiation-induced upper aerodigestive stenosis causing dysphagia and stridor were excluded. Non-English articles were also excluded. The search terms in the database included dysphagia, stridor, pharyngeal stenosis and oesophagal stenosis and radiation complication on the head and neck. The abstracts of the published articles were identified by this search method and other articles were identified manually from citations. This manuscript reviews the epidemiology, pathophysiology, clinical outcome, investigations and treatment of the radiation-induced dysphagia and stridor. This review article will surely act as a baseline for further prospective studies of radiation-induced dysphagia and stridor by pharyngeal or upper aerodigestive stenosis could be designed and helps as a spur for further research in this dreaded clinical entity.

184 abstracts identified through PubMed, Scopus, Google Scholar and Medline database with the word dysphagia and stridor following radiotherapy in head and neck cancer, pharyngeal stenosis, esophageal stenosis, radiotherapy in head and neck cancer and complications.

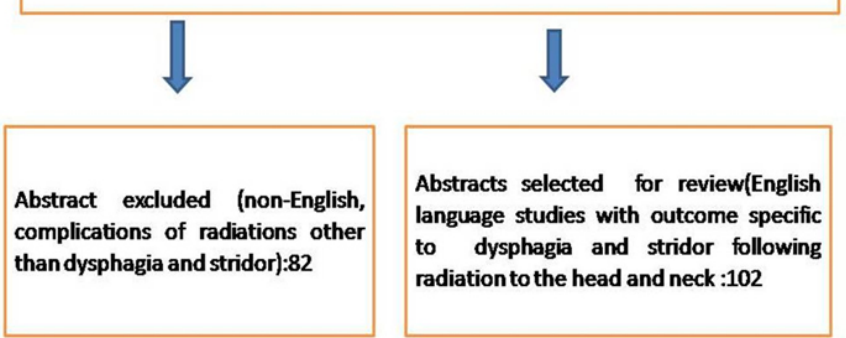

Figure 1: Methodology for literature search

\section{EPIDEMIOLOGY}

Head and neck cancer survivors are living longer because of the epidemiological changes in cancer and advancement in the treatment. However, clinicians encounter a growing number of patients with late effects of radiotherapy. Radiation associated dysphagia which progresses throughout the years after head and neck cancer treatment is often challenging in the era of organ preservation. ${ }^{4}$ The epidemiological studies of radiation-induced dysphagia and stridor are less documented in the medical literature. Dysphagia and stridor are extremely rare and morbid complaint by the patients following radiation therapy in nasopharyngeal carcinoma or any head and neck squamous cell carcinoma. ${ }^{5}$ Despite the hazardous effect of dysphagia and stridor on quality of life, the prevalence of this pharyngeal or oesophagal stenosis in patients who had radiated as part of their treatment for head and neck malignancy remains unclear. Nasopharyngeal carcinoma is commonly found in southern China and Hong Kong. ${ }^{6}$ The incidence of the nasopharyngeal carcinoma is $20 / 100,000$ population in a year at a high-risk region. ${ }^{7} \mathrm{Al}-$ though uncommon, the late side effects like dysphagia and stridor are morbid complaint by radiotherapy in nasopharyngeal carcinoma or any head and neck squamous cell carcinoma. Radiotherapy is the treatment of choice in patients with nasopharyngeal carcinoma without distant metastasis. There were different late complications of radiation therapy documented in that region such as endocrine dysfunction and injury to the temporal lobe. ${ }^{8}$ The nasopharyngeal carcinoma is mainly treated by radiotherapy and it needs to follow up based on clinical, endoscopic radiological monitoring. The curative doses of radiotherapy in nasopharyngeal carcinoma can lead to complications like choanal stenosis and pharyngeal stenosis. The pharyngeal stenosis can cause dysphagia and severe variety of manifests stridor. Only a few such cases are reported in the medical literature and this may be a lack of follow up of these patients.

\section{PATHOPHYSIOLOGY}

The exact pathophysiology for radiation-induced pharyngeal stenosis is not well understood. The pathophysiology for dysphagia and stridor following radiotherapy to the head and neck area is complex. Radiotherapy induced xerostomia may induce the damage of the salivary glands, esophagitis and tracheobronchial aspiration which lead to stenosis of the pharynx and oesophagus. ${ }^{9}$ It is believed that radiation exposure to the neck causes fibrosis, gradual reduction of the vascularity and denervation atrophy of the pharyngeal muscles and mucosa leads to the pharyngeal stenosis and the severity is associated with the dose of radiation and field of radiation. In the affected area of the radiation-induced fibrosis, there is an increased proliferation of the fibroblasts, infiltration of the inflammatory cells and increased amount of the extracellular matrix. ${ }^{10}$ Radiotherapy alone or combined with chemotherapy in head and neck cancer may induce pharyngeal or oesophagal stenosis despite the preservation of the organ. ${ }^{11}$ The muscles of the pharynx, oesophagus and larynx are found deep to the submucosal and overlaid by stratified squamous epithelium and the lamina propria of the mucosal layer. The seromucous glands and lymphoid aggregates are seen throughout the mucosal layer and so radiation injury to these areas lead to noticeable soft tissue deformities like stenosis or stricture by altering the contour of the pharynx, larynx and oesophagus. Despite advancement in radiotherapy technique, there are still associated complications of radiation and these include mucositis, dermatological changes, swallowing defects due to changes in pharynx and oesophagus. These manifestations are found at the time of acute clinical period, the first 6 months after radiotherapy. ${ }^{12}$ 
Head and neck squamous cell carcinomas are common types of malignancies found in the population. The treatment options for squamous cell carcinoma of the head and neck region are usually surgery and chemoradiation. The radiotherapy and concomitant chemotherapy are widely accepted treatment options for organ preservation. ${ }^{13}$ There are considerable shuffling of the treatment options by the clinicians for treating the head and neck cancer. Some surgeon chooses preoperative radiotherapy, while some prefer postoperative radiation therapy. In a few cases, full therapeutic doses of radiation are given, although in the reduced dose. However, clinicians should know about the benefits of combinations of these two treatment modalities. The upper aerodigestive tract of the head and neck region is highly susceptible to radiation-induced damage and cause stenosis of the pharynx with impairment of the swallowing. ${ }^{14}$ The chemotherapy for malignancy of the head and neck region may increase the chance of stenosis of the upper aerodigestive part in cancer patients as adjuvant chemotherapy has been found to increase the chance of stricture formation in patients of oesophagal cancer. ${ }^{15}$ The benign hypopharyngeal or oesophagal fibrosis is usually secondary to the fibrosis at the lamina propria and sub-mucosa although inflammation, oedema and spasm of the muscles play important role in the etiopathogenesis of stricture formation. One study reported that absorbed dose of the radiation to the cricopharyngeal muscle and cervical part of the oesophagus plays a vital role in causing dysphagia. ${ }^{16}$ The resultant structure cause temporary or permanent dysmotility. Ulceration at the hypopharynx or oesophagus may also increase the chance of stricture formation. It has been documented that oesophagal stricture can occur up to 5 years after completion of the radiotherapy. ${ }^{17}$

The introduction of intensity-modulated radiation therapy (IMRT) in the treatment of the head and neck cancer greatly minimized the damage to the adjacent part and adjust maximum to the target tissue. However, the loco-regional control of the malignancy of the head and neck region needs aggressive radiation treatment for reducing the tumour cells. ${ }^{18}$ These increased radiation schedules and high doses of the radiation (60 to 70 Gray), leading to rapid dose accumulation which is less tolerable. ${ }^{19}$

\section{CLINICAL PRESENTATIONS}

Stenosis at the hypopharynx or upper aerodigestive tract is an uncommon late complication of the radiotherapy for head and neck cancer. Severe radiation exposure to the patients is often prone to such complication, which may be found months to years after radiotherapy. Although the technique of radiotherapy in head and neck cancer is offering a promising treatment outcome, the complications during or after irradiation are inevitable and the quality of life after radiotherapy is usually compromised. The strictures of the hypopharynx, larynx and cervical part of the oesophagus are often complications of the radiotherapy. The cervical part of the oesophagus is usually included in the lower margin of the radiotherapy portal for head and neck cancer. The severity of the clinical presentations is closely related to the degree of the stenosis. The symptoms occurred due to the obstruction of the pharynx. The obstruction of the pharynx leads to dysphagia and breathing difficulty. Severe stenosis of the pharynx may lead to compromised airway and lead to stridor. ${ }^{20}$ Patients may also present with symptoms due to radiation effects on the other area of the head and neck such as dry mouth and impairment of hearing because of the eustachian dysfunction. ${ }^{21}$ The diagnosis of the pharyngeal stenosis is often late as patients are usually asymptomatic in the initial period after radiotherapy. So, follow up endoscopic examinations should be done at regular interval. Patients with postradiotherapy hypopharyngeal and oesophagal stenosis or strictures initially show dysphagia to the solid foods and then progresses to the liquid foods over time. The degree or severity of the dysphagia is associated with the size of the lumen of the pharynx and oesophagus. In the case of oesophagal stricture, the diameter less than $12 \mathrm{~mm}$ causes dysphagia to the normal diet. Patients may also present with chest or abdominal pain, regurgitation of food, aspiration of food and weight loss if the stenosis is found at the upper oesophagus.

\section{INVESTIGATIONS}

The diagnosis of the pharyngeal stenosis should be properly evaluated with help of the fiberoptic nasopharyngolaryngoscopy and computed tomography (CT) scan of the neck. Fiberoptic nasopharyngolaryngoscopy or rigid nasal endoscopy is helpful to assess the site and severity of the stenosis (Figure 2).

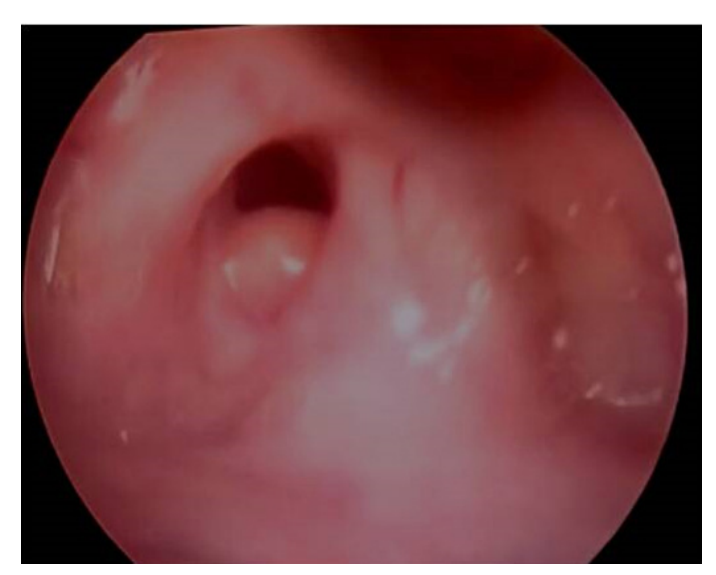

Figure 2: Fiberoptic nasopharyngolaryngoscopy showing radiation induced stenosis at the hypopharynx.

Endoscopic examination reveals the anatomical location of the obstruction and confirms whether the lumen is obstructed completely or not. CT scan allows the confirmation and quantifying the degree of the stenosis (Figure 3). 


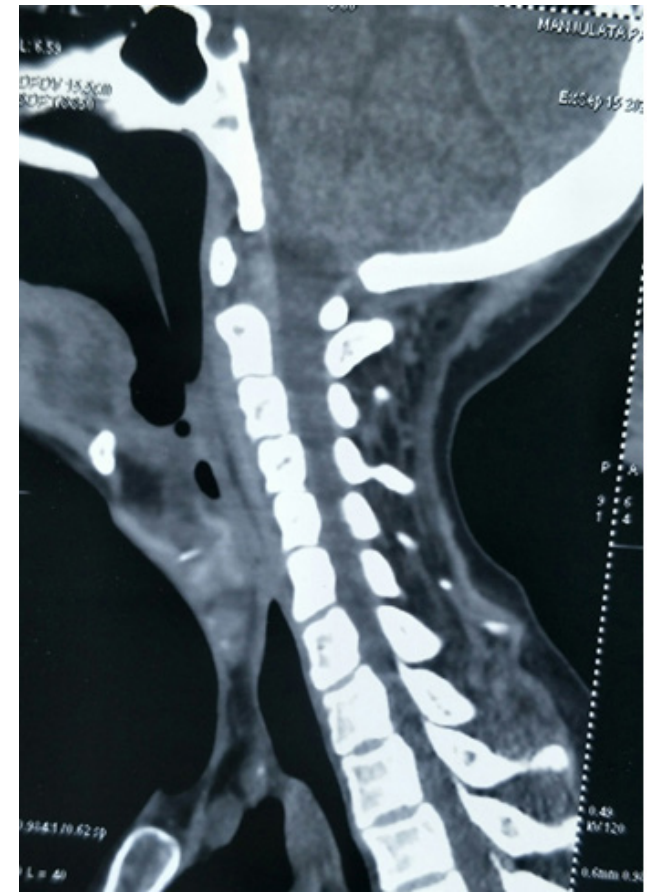

Figure 3: CT scan of the neck showing radiation induced stenosis at the upper aerodigestive tract.

Direct laryngoscopy and biopsy should be mandatory from the stenosis site for excluding any residual or recurrent neoplasm and to assess the characteristics of the stricture. If the stenotic site is narrow and irregular the pediatric endoscope can be used for proper evaluation and biopsy. ${ }^{22}$ Stenosis at the hypopharynx is frequently found following total laryngectomy. Radiological examination often shows the narrowing at the superior surgical closure sites. Anastomotic strictures are seen after hypopharyngeal reconstruction in resection of the hypopharyngeal malignancy. ${ }^{23}$

\section{TREATMENT}

The treatment of the pharyngeal or oesophagal stenosis following radiotherapy to malignancy of the head and neck area is often challenging because of the high incidence of recurrence. Stenosis at the upper aerodigestive tract may require dilatation or nasogastric tube feeding depending based on the severity of the obstruction. Surgical intervention is the treatment of choice for pharyngeal or oesophagal stenosis. There are many techniques available for correction of the pharyngeal stenosis. The techniques include simple dilatation, excision of the scar tissue, resection of the scar tissue with the use of rotational mucosal flaps, use of stent and coblation excision of the scar tissue and topical injection of the mitomycin-C injection. Patients often referred to the gastroenterologist for therapeutic oesophagal of pharyngeal dilatations if the stenosis is confirmed. If patients again complain of dysphagia after dilatation, repeat dilatation is advised. The endoscopic dilatation is the first line of treatment for the pharyngeal and oesophagal strictures. The dilatation should not be forceful as there is the risk of perforation. The first dilatation of the stenosis was reported in the seventh century with help of carved whalebone. ${ }^{24}$ and the several dilators have been developed. Mercury-filled Maloney dilators are flexible bougies without guide wires are useful to dilate the benign strictures. ${ }^{24}$ Balloon dilators are a useful technique for a successful outcome but more costly and may not be used more than once. The microdebrider is also a good option for excision of the scar tissue from the pharyngeal stenosis. The major challenge of this procedure is re-stenosis. The timing of the repair is important in case of pharyngeal stenosis. Early intervention can cause re-stenosis as scar bands which continue to contract and obstruct the pharynx. The surgeons those do not keep postoperative stenting often perform serial dilatations or revision endoscopic excision or removal of the crusting. ${ }^{25}$

After dilation of the stenotic segment, the pharyngeal wall at the site of the widening can be applied with mitomycinC. Mitomycin-C is an antineoplastic antibiotic which acts as an alkylating agent and inhibits protein, DNA synthesis and fibroblast proliferation. Mitomycin-C is an antibiotic produced from Sterptomyces caespitosus..$^{26,27}$ Mitomycin-C gives its effect by cross-linking to the DNA at the cellular level. It does not act randomly, rather it acts preferentially bind to the genes that are being induced. The Mitomycin-C is safe and used topically at various surgical locations for preventing re-stenosis without any major side effects. ${ }^{28}$ It also prevents the chance of synechia formation at the upper aerodigestive tract after dilatation or surgery with coblation. Although Mitomycin-C is a toxic drug, there has been no report regarding systemic toxicity. ${ }^{29}$ The safety dose of Mitomycin is topically $0.4 \mathrm{mg} / \mathrm{ml}$ in the treatment of the pharyngeal stenosis. ${ }^{29}$

Currently use of the radiofrequency or coblation for excision of the scar tissue with application of mitomycin is providing effective result with less chance of re-stenosis. ${ }^{30}$ Coblation technology uses bipolar radiofrequency energy which generates a field of ionized sodium molecules that ablates and coagulates soft tissues without any thermal injury. Conductive saline solution is kept in the gap between the soft tissue and device tip and converts the saline into the ionized plasma layer. One this plasma layer touches the tissue, there is enough break the molecular bonds, causing molecular dissociation. This effect of the tissue dissociation occurs at the temperature of about $4^{\circ} 0 \mathrm{C}$ to $70^{\circ} \mathrm{C}$, so reduce the thermal damage to the tissue. ${ }^{31,32}$

The complications of the above procedures are minimal. After procedures of dilatations, slight bleeding and erosions of the mucosal linings are commonly found and these manifestations spontaneously resolve without any specific treatment. However, there are two important life-threatening complications may be found such as perforation of the pharynx or oe- 
sophagus and hematemesis. Post-dilatation check-up should be done by endoscope to rule out such complications. In the case of severing hypopharyngeal stenosis, the airway is also compromised which leads to stridor and this life-threatening situation need emergency tracheostomy for securing the airway. After successful dilation of hypopharyngeal stenosis, the tracheostomy tube can be decannulated.

\section{CONCLUSION}

Radiotherapy is the treatment of choice in nasopharyngeal carcinoma without distant metastasis and also play an important role in the treatment of other head and neck squamous cell carcinoma. Radiation-induced biological alteration after radiation therapy to the head and neck area can be undetected for years but eventually lead to functional deficits by causing pharyngeal or oesophagal stenosis. In the early stage of the complications, patients may be asymptomatic but once the stenosis develops in the upper aerodigestive tract, leads to dysphagia and stridor. It minimizes the quality of life and increases the incidence of mortality even in the cancer-free survivor. Although there is no effective treatment available, dilatation of the pharynx or oesophagus with help of the different technique like bougies, microdebrider and coblation are treatment options. Clinicians should be aware of the radiation-induced complications leading to pharyngeal stenosis and its severity along with its treatment options.

\section{Conflict of interest: Nil}

Funding: No Funding sources were granted or used specifically for this work.

\section{Author Contribution:}

SKS: Concept, data collection and data analysis;

SA: Data collection, data analysis, and drafting the manuscript;

SL: Data collection and drafting.

\section{REFERENCES}

1. Morgan HE, Sher DJ.Adaptive radiotherapy for head and neck cancer. Head Neck Cance 2020;5:1-6.

2. Swain SK, Samal S, Mohanty JN, Choudhury J. Nasopharyngeal carcinoma among the pediatric patients in a non-endemic region: our experience at a tertiary care teaching hospital in eastern India. Egy Pediatr Assoc Gazz 2020;68:23.

3. Lam KS, Tse VK, Wang C, Yeung RT, Ho JH. Effects of cranial irradiation on hypothalamic-pituitary function- a 5-year longitudinal study in patients with nasopharyngeal carcinoma. Q J Med 1991;78:165-176.

4. De Felice F, de Vincentiis M, Luzzi V, Magliulo G, Tombolini $\mathrm{M}$, Ruoppolo $\mathrm{G}$, et al. Late radiation-associated dysphagia in head and neck cancer patients: evidence, research and management. Oral Oncol 2018;77:125-130.
5. Swain SK, Samal S, Anand N, Mohanty JN. Pediatric nasopharyngeal carcinoma. Int J Health Allied Sci 2019; 9:1-6.

6. Swain SK, Samal S, Sahu MC.Nasopharyngeal carcinoma in a six-year-old female child: a case report. Pediatria Polska-Polish J Paediatr 2019; 94(2):132-135.

7. Sun LM, Li CI, Huang EY, Vaughan TL. Survival difference by race in nasopharyngeal carcinoma. Am J Epidemiol 2007; 165:271-278.

8. Lam KS, Tse VK, Wang C, Yeung RT, Ho JH. Effects of cranial irradiation on hypothalamic-pituitary function - a 5-year longitudinal study in patients with nasopharyngeal carcinoma. Q J Med 1991;78:165-176.

9. Pauloski BR, Rademaker AW, Logemann JA, et al. Swallow function and perception of dysphagia in patients with head and neck cancer. Head Neck 2002;24:555-565.

10. Sciubba JJ, Goldenberg D. Oral complications of radiotherapy. Lancet Oncol 2006; 7:175-183.

11. Sullivan CA, Jaklisch MT, Haddad R, et al. Endoscopic management of hypopharyngeal stenosis after organ sparing therapy for head and neck cancer. Laryngoscope 2004;114:1924-1931.

12. Strauss M. Long term complication of radiotherapy confronting the head and neck surgeon. Laryngoscope 1983;93:310-313.

13. Kuo P, Chen MM, Decker RH, Yarbrough WG, Judson BL. Hypopharyngeal cancer incidence, treatment, and survival: temporal trends in the United States. Laryngoscope 2014; 124(9):2064-2069.

14. Batth SS, Caudell JJ, Chen AM. Practical considerations in reducing swallowing dysfunction following concurrent chemoradiotherapy with intensity-modulated radiotherapy for head and neck cancer. Head Neck 2014;36(2):291-298.

15. Seeman H, Gates J, Morris T. Esophageal dysfunction years after radiation therapy. Dig Dis Sci 1992;37:303-306.

16. Alterio D, Gerardi MA, Cella L, Spoto R, Zurlo V, Fodor C. Radiation-induced acute dysphagia. Strahlentherapie Oncol 2017;193(11):971-981.

17. Moody GA, Probert CSJ. Mercury bougie self-dilation of the oesophagus in the 1990s. J Clin Gastoenterol1992;15:264-273.

18. Fenwick JD, Pardo-Montero J, Nahum AE, Malik ZI. Impact of schedule duration on the head and neck radiotherapy: accelerated tumour repopulation versus compensatory mucosal proliferation. Int J Radiat Oncol Biol Phys 2012;82(2):1021-1030.

19. Peters LJ, Ang KK, Thames HD Jr. Accelerated fractionation in the radiation treatment of head and neck cancer. A critical comparison of different strategies. Acta Oncol 1988;27(2):185-194.

20. Swain SK, Mohanty S, Singh N, Samal R. An unusually Giant Hematoma threatening to the laryngeal airway. Int J Otorhinolaryngol Clin 2014;6(3):92-94.

21. Swain SK, Janardan S, Mohanty JN. Endoscopy guided eustachian tube balloon dilation: Our experiences. Iran J Otorhinolaryngol 2020 Sep;32(112):287-294.

22. Ferguson DD. Evaluation and management of benign oesophagal strictures. Dis Esophagus 2005;18:359-364.

23. Annino DJ, Goguen LA. Mitomycin C for the treatment of pharyngoesophageal structures after total laryngopharyngectomy and microvascular free tissue reconstruction. Laryngoscope 2003;113:1499-1502.

24. Spiess AE, Kahrilas PJ. Treating achalasia: from whalebone to laparoscope. JAMA 1998 280:638-642

25. Van Den Abbeele T, Francois M, Narcy P. Transnasal endoscopic treatment of choanal atresia without prolonged stenting. Arch Otolaryngol Head Neck Surg 2002;128:936-940.

26. Prasad M, Ward RF, April MM, Bent JP, Froehlich P. Topical mitomycin as an adjunct to choanal atresia repair. Arch Otolaryngol Head Neck Surg 2002;128:398-400. 
27. Lenka S, Swain SK, Bhuyan R, Sahu MC.Fungal infection in the oral cavity: A Review. Int J Cur Res Rev.2020;12(18):149-153.

28. Kim KR, Song HY, Shin JH, Kim JH, Choi EK, Yang ZQ, et al. Efficacy of mitomycin-C irrigation after balloon dacryocystoplasty. J Vasc Interv Radiol 2007; 18(6):757-762.

29. Rosseneu S, Afzal N, Yerushalmi B, Ibarguen-Secchia E, Lewindon $\mathrm{P}$, Cameron D, et al. Topical application of mitomycin-C in oesophageal strictures. J Pediatr Gastroenterol Nutr 2007; 44:336-341.

30. Hussein J, Tan TS, Chong AW, Narayanan P, Omar R. Velopharyngeal and choanal stenosis after radiotherapy for naso- pharyngeal carcinoma. Auris Nasus Larynx 2013;40(40):323326.

31. Swain SK, Ghosh TK, Munjal S, Mohanty JN. Microscope assisted coblation tonsillectomy among paediatric patients-our experiences at an Indian teaching hospital. Pediatr Pol 2019; 94(3):170-174.

32. Samal S, Debta P, Swain SK.Role of chromium enriched tobacco in the occurrence of oral carcinogenesis. Int J Cur Res Rev 2020;12(18):20-24. 